\title{
UNA REVISIÓN CRÍTICA DE LA PRÁCTICA DE DISEÑO POR SISMO DE ESTRUCTURAS EN MÉXICO
}

\author{
Mario E. Rodríguez ${ }^{(1)}$
}

\begin{abstract}
RESUMEN
Se evalúan las demandas de aceleraciones y desplazamientos en los terremotos de México 1985, Chile 2010, y Christchurch 2010 y 2011. Se encontró que en todos los casos estas demandas fueron mayores que las de normativas de diseño existentes. Se compararon la respuesta ante terremotos de edificaciones típicas en la ciudad de México en 1985 y en Concepción, Chile 2010, y se encontró un mejor comportamiento en sistemas estructurales que utilizan muros con respecto a aquellos basados marcos. Con base en esta investigación se sugieren cambios en la práctica de diseño por sismo de estructuras en México.
\end{abstract}

Palabras clave: respuesta sísmica; marcos; muros estructurales; concreto reforzado

\section{A CRITICAL REVIEW OF THE SEISMIC DESIGN PRACTICE OF STRUCTURES IN MEXICO}

\begin{abstract}
This research studies acceleration and displacement response spectra using ground motions recorded in past earthquakes, such as Mexico 1985, Chile 2010 and Christchurch 2010 and 2011. These calculated demands were larger than those specified by building codes. The seismic response of typical buildings during the studied earthquakes was compared with the observed behavior. The results showed a better seismic behavior of structural walls as compared with that of frames. Findings from this study are used for suggesting changes in the seismic design practice in Mexico.
\end{abstract}

Keywords: earthquake response; frames; structural walls; reinforced concrete

\section{INTRODUCCIÓN}

En el diseño por sismo de estructuras en México, así como en otros países, es usual para fines de diseño el emplear espectros elásticos de aceleraciones, a partir de los cuales se obtienen espectros de diseño elásticos de desplazamientos. Debido al alto costo de soluciones estructurales, si se diseñaran para un comportamiento elástico, la práctica general de diseño sísmico es el diseñar estructuras con resistencias menores que las elásticas; sin embargo, como se muestra en esta investigación, las revisiones de demanda y capacidad de desplazamiento de la estructura empleando espectros de diseño no son consideradas de manera adecuada por normativas de construcción. En este trabajo se muestra que estos espectros de diseño, aunque resultan del empleo de modelos teóricos específicos, no necesariamente permiten conocer de manera confiable los valores de aceleraciones y desplazamientos espectrales que se obtendrían como

Artículo recibido el 6 de octubre de 2015 y aprobado para su publicación el 12 de junio de 2016. Se aceptarán comentarios y/o discusiones hasta cinco meses después de su publicación.

${ }^{1}$ Investigador, Instituto de Ingeniería, UNAM, Ciudad Universitaria, CP 04510, México DF, mrod@unam.mx 
resultado de un terremoto importante durante la vida útil de la estructura. Tampoco estas normativas transmiten al ingeniero la importancia de considerar las posibles demandas de desplazamientos en edificios en un terremoto, así como posibles soluciones para el control de éstos. En esta investigación se emplean registros de aceleraciones obtenidos en tres terremotos en el mundo, cuyas ordenadas espectrales superaron por mucho las especificadas por normativas para el diseño de estructuras que respondieron a estos terremotos. Estos terremotos fueron los experimentados en la Ciudad de México en 1985, Concepción en Chile 2010, y Christchurch en Nueva Zelanda 2011.

\section{PRÁCTICAS DE NORMATIVAS DE DISEÑO POR SISMO PARA LA CARACTERIZACIÓN DEL SISMO DE DISEÑO Y DE LA RESPUESTA SÍSMICA DE ESTRUCTURAS}

\section{Caracterización del sismo de diseño}

Para la caracterización del sismo de diseño es relevante la estimación de terremotos y el consecuente peligro sísmico. La ubicación, magnitud y el intervalo de recurrencia de los terremotos son parámetros altamente inciertos, como se ha observado en terremotos, destacando por ejemplo el caso de los terremotos de Christchurch en Nueva Zelanda en 2010 y 2011. Usualmente, estos parámetros y sus incertidumbres son estimados empleando modelos probabilistas. De acuerdo con Wang (2015), para un terremoto de magnitud media, $M$, la probabilidad anual de excedencia de la aceleración máxima del sitio, $v(a)$, se obtiene como el producto de la probabilidad de excedencia anual del terremoto, $P E_{E}$, y la probabilidad de excedencia de un registro de aceleraciones dado, $P E_{a}$, es decir:

$v(a)=P E_{E} P E_{a}$

donde $P E_{E}$ en un año se estima como (Wang, 2015):

$P E_{E}=1-e^{-1 / \tau} \approx \frac{1}{\tau}$

donde $\tau$ es el periodo de recurrencia medio del terremoto.

El valor de $P E_{E}$ depende de la ubicación, magnitud e intervalo de recurrencia del terremoto, los cuales por no tener características deterministas, se emplean modelos probabilistas.

El valor de $P E_{a}$ depende además de las características del terremoto, de leyes de atenuación y de efectos de sitio, y es un problema también probabilista, con altas incertidumbres. El modelo de atenuación es importante; sin embargo, la incertidumbre puede ser muy alta. Por ejemplo, el Manual de Diseño por Sismo de la CFE (CFE, 2008) define ordenadas espectrales de diseño cuyos periodos de retorno pueden cambiar de manera significativa dependiendo del modelo de distribución de probabilidades que se emplee para las leyes de atenuación. En un cierto periodo estructural, para un valor dado de la aceleración espectral, el periodo de retorno puede cambiar de 200 a 500 años, dependiendo de si se emplea distribuciones lognormales convencionales o truncadas, respectivamente (CFE, 2008).

\section{Caracterización de la respuesta sísmica de estructuras}

Para caracterizar la respuesta de estructuras, los procedimientos de normativas de diseño por sismo hacen énfasis en la definición de la resistencia lateral de la estructura, con lo que la comparativa de las demandas y capacidades de distorsiones en la estructura pasan a un segundo plano. Sin embargo, se ha observado en diversos terremotos en el mundo, que en general las estructuras alcanzan su resistencia lateral, a partir de la cual su respuesta es inelástica. Si esta respuesta se mide en función de 
desplazamientos laterales de la estructura, o mejor aún en función de las llamadas distorsiones globales (cociente del desplazamiento de azotea y altura del edificio) o de entrepiso (en esta investigación se emplea la distorsión global), es deseable que la demanda de distorsiones sean menores que su capacidad. Además de algunas características deseables en una estructura como su regularidad, un factor relevante para aumentar esta capacidad, y que puede ser controlado por el ingeniero, es lograr un detallado adecuado del acero de refuerzo. Cuando este detallado es deficiente, lo que ocurre generalmente en estructuras de ductilidad limitada ( $Q=2$ en el caso de México), en un terremoto las secciones críticas del elemento estructural requerirían una capacidad de deformación mayor que la que es posible en estas secciones críticas. Esto eventualmente podría llevar al colapso de la estructura, incluso con modos de falla frágiles, como ha sido observado en elementos de concreto reforzado cuando en sus secciones críticas se suelda el acero de refuerzo longitudinal (Rodríguez y Torres, 2013), práctica que se permite en México. En opinión del autor, un análisis de la relación costo-beneficio, incluyendo el costo de la vida humana, de un sistema estructural adecuado que controle desplazamientos y diseñado con detalles de refuerzo favorables para un buen comportamiento sísmico podría mostrar la conveniencia del empleo de este sistema estructural y detallado.

En esta investigación se muestran ejemplos que indican la necesidad de mejoras en los procedimientos de normativas de diseño para caracterizar el sismo de diseño y para caracterizar la respuesta sísmica de estructuras. En lo que sigue se empieza revisando el problema de caracterización del sismo de diseño, para lo cual se emplean las ordenadas espectrales de registros de aceleraciones típicos obtenidos en los terremotos en la Ciudad de México en 1985, Concepción en Chile 2010, y Christchurch en Nueva Zelanda 2010 y 2011, y se comparan con los valores especificados por normativas de construcción en los lugares de los terremotos estudiados.

\section{COMPARATIVA DE DEMANDAS SÍSMICAS OBSERVADAS Y ESPECIFICADAS EN ALGUNOS TERREMOTOS OCURRIDOS EN EL MUNDO}

\section{Terremotos en Christchurch en Nueva Zelanda en 2010 y 2011}

En Febrero 22, 2011, en horas laborables, un terremoto afectó la ciudad de Christchurch en Nueva Zelanda, el epicentro prácticamente se ubicó en la ciudad y su magnitud fue $M_{w}=6.2$. Este terremoto se puede considerar una réplica del terremoto ocurrido el 4 de Septiembre, 2010, con epicentro a una distancia de alrededor de $40 \mathrm{~km}$ de la ciudad, con $M_{w}=7.1$ (Bradley et al, 2014). El terremoto de 2011 causó colapsos y daños significativos en edificios residenciales y comerciales, viviendas, puentes y líneas subterráneas vitales, así como 185 muertes. Estos terremotos ocurrieron en fallas del terreno no conocidas por los expertos, y antes de estos terremotos se consideraba que Christchurch no se ubicaba en una región de alta sismicidad (EERI, 2011). Se contó con información de alrededor 20 estaciones de acelerómetros en la ciudad (Bradley et al, 2014), de estas estaciones se seleccionó para esta investigación la estación CCCC, en la zona de mayor daño en la ciudad. La estación se ubica en el tipo de suelo D, suelos blandos o profundos, de la clasificación de suelos especificada en la norma New Zealand Loading Standard (NZLS, 2004), los valores de aceleración y velocidad máxima del terreno (por su siglas en inglés PGA y PGV) registrados en esta estación fueron $0.43 g$ y $0.56 \mathrm{~m} / \mathrm{s}$, y la distancia del plano de falla a la estación es sólo $2.8 \mathrm{~km}$ (Bradley et al, 2014). La Tabla 1 resume algunas características de los terremotos mencionados de 2010 y 2011 y de los registros de aceleraciones. 
Tabla 1. Características de registros de aceleraciones empleados en esta investigación

\begin{tabular}{|c|c|c|c|c|c|c|c|c|}
\hline Nombre de la estación & Código & Fecha & $\begin{array}{l}\text { Magnitud } \\
(M w)\end{array}$ & Dirección & $\begin{array}{l}P G A \\
(g)\end{array}$ & $\begin{array}{l}P G V \\
(\mathrm{~m} / \mathrm{s})\end{array}$ & $\begin{array}{l}\text { Tipo de suelo } \\
\text { ASCE 7-10 }\end{array}$ & $\begin{array}{l}V_{s 30} \\
(\mathrm{~m} / \mathrm{s})\end{array}$ \\
\hline \multirow{2}{*}{$\begin{array}{c}\text { Christchurch Cathedral } \\
\text { College }\end{array}$} & $\mathrm{CCC}$ & 4 Sept 2010 & 7.1 & N64E, N26W & 0.22 & 0.54 & $D^{1}$ & - \\
\hline & $\mathrm{CCC}$ & 22 Feb 2011 & 6.2 & N64E, N26W & 0.43 & 0.56 & $D^{1}$ & - \\
\hline Concepcion Centro & CON & 27 Feb 2010 & 8.8 & L, T & 0.39 & 0.67 & D & 230 \\
\hline Concepcion San Pedro & CCSP & 27 Feb 2010 & & NS, EW & 0.65 & 0.45 & C & 390 \\
\hline SCT & $\mathrm{SCT}$ & 19 Sept 1985 & $\begin{array}{l}8.1 \\
(\mathrm{Ms})\end{array}$ & NS, EW & 0.17 & 0.61 & Suelo Blando & - \\
\hline
\end{tabular}

${ }^{1}$ Clasificación de las NZLS (2004)

Las figuras 1a y $1 \mathrm{~b}$ muestran espectros de aceleraciones y desplazamientos elásticos para la estación CCCC en los terremotos de 2010 y de 2011, en sus dos componentes horizontales, y fueron calculados con un porcentaje del amortiguamiento crítico igual a 5\%. También las figuras 1a y $1 \mathrm{~b}$ muestran con líneas negras continuas los espectros de diseño elásticos especificados por las NZLS (2004) para esta parte de la ciudad, tanto el correspondiente al periodo de retorno de 500 años, como el de 2500 años (EERI, 2011). Los resultados de las figuras 1a y 1b muestran que las ordenadas espectrales elásticas calculadas para el terremoto de 2011 son bastante mayores que las del terremoto de 2010 en la zona de periodos estructurales, $T$, menores que $1.5 \mathrm{~s}$, mientras que para periodos mayores que ese valor las diferencias de ordenadas espectrales para ambos terremotos tienden a ser menores. Aproximadamente, las ordenadas espectrales de los registros obtenidos en el terremoto de 2010 se acercan a las ordenadas especificadas por la NZLS (2004) para el terremoto con periodo de retorno de 500 años, mientras que las ordenadas espectrales de registros obtenidos en el terremoto de 2011 se acercan o incluso son mayores que las ordenadas especificadas por la NZLS (2004) para el terremoto con periodo de retorno de 2500 años.

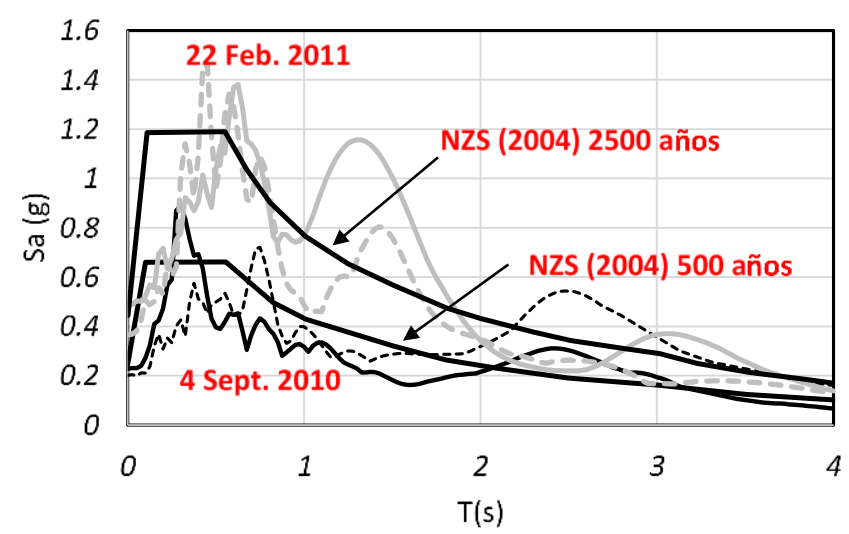

(a) Aceleraciones espectrales horizontales obtenidas con registros de la estación Christchurch Cathedral College en los terremotos de Christchurch 2010 y 2011 


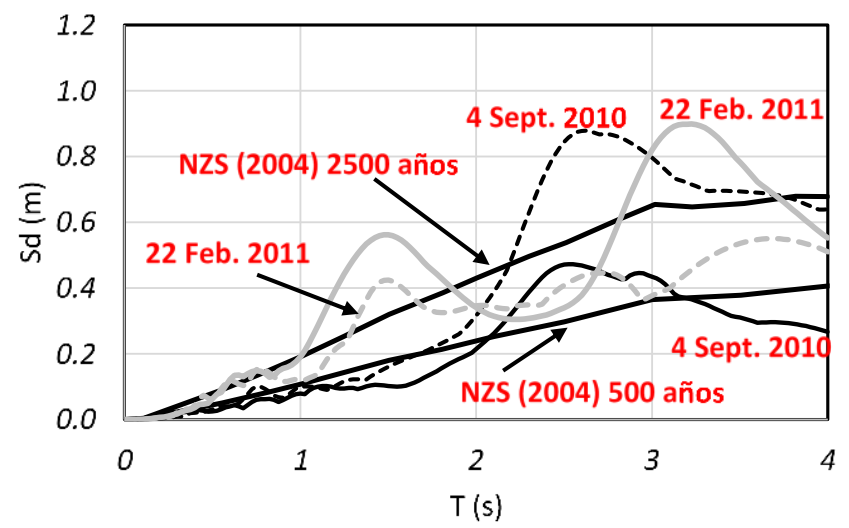

(b) Desplazamientos espectrales horizontales obtenidos con registros de la estación Christchurch Cathedral College en los terremotos de Christchurch 2010 y 2011

Figura 1. Aceleraciones y desplazamientos espectrales horizontales obtenidos con registros de aceleraciones de los terremotos de México 1985; Maule, Chile 2011, y Christchurch 2010 y 2011

(Continuación)

Llama la atención el hecho de que en un tiempo menor que un año ocurrieron los terremotos de periodos de retorno de 500 y 2500 años especificados por la NZLS (2004). Estos eventos sugieren la revisión de los procedimientos probabilistas que se emplean para la estimación del peligro sísmico de un lugar. Por ejemplo, con base en estos eventos sísmicos en Christchurch, se ha propuesto un nuevo modelo de estimación de las ordenadas espectrales de diseño para un lugar, en el cual durante por ejemplo un tiempo medio de 50 años se considera que estas ordenadas espectrales van cambiando con el tiempo (Gerstenberger et al., 2014).

\section{Terremoto de Maule, en Concepción, Chile, 27 Febrero 2010, $M_{w}=8.8$}

El terremoto de Maule en febrero 2011, de magnitud 8.8, así como el de Tohoku, Japón, el 11 de marzo 2011, de magnitud 9, son los eventos de mayor magnitud con un número considerable de registros de aceleraciones del terreno. En el terremoto de Maule se obtuvieron registros de aceleraciones en 31 estaciones (Boroschek et al., 2012) que contribuyen al conocimiento de terremotos en zonas de subducción. El hipocentro de este terremoto se ubicó bajo el mar a una distancia de $60 \mathrm{~km}$ Nor-Oeste de la ciudad de Concepción, una de las principales ciudades en Chile. La longitud de ruptura que causó el terremoto fue de alrededor de $500 \mathrm{~km}$. Los daños fueron mayores en estructuras altas y nuevas, con un solo colapso (Edificio de Alto Río), con ocho muertos en ese edificio. El mayor número de muertes fue resultado del maremoto que causó el terremoto. En la población de edificios de concreto reforzado de 9 o más niveles construidos después de 1985 (año del anterior terremoto importante en Chile), el daño severo fue en el 2\% de esta población (Massone et al., 2012). Antes de 1985 la mayoría de edificios tenían menos de 15 niveles, si se suma esta población a la anteriormente mencionada, el referido porcentaje de daños es bastante menor (Massone et al., 2012). La mayoría de edificios dañados tanto en Concepción como en Viña del Mar tenían un periodo fundamental efectivo cercano a 1s (Bonelli, 2015). Como se muestra más adelante, estructuras con periodo fundamental cercanos al valor mencionado, habrían tenido desplazamientos laterales a nivel azotea menores que uno por ciento de la altura del edificio.

Los edificios típicos chilenos tienen cantidad y área de muros estructurales de concreto reforzado (CR), bastante mayores que las que típicamente tienen edificios en México. Un parámetro que cuantifica esta participación de muros es la relación $A_{w} / A_{f}$, donde $A_{w}$ es el área de muros estructurales en una 
dirección, y $A_{f}$ es el área de la planta del edificio. Estadísticas de edificios de con muros estructurales de CR construidos en Santiago en el periodo1939 a 2006 muestran que el 78\% de estos edificios tienen relaciones $A_{w} / A_{f}$ entre $1.5 \%$ y $3.5 \%$ (Massone et al., 2012). La tendencia en la construcción de edificios después de 1985 fue la de emplear edificios no sólo más altos, sino también más esbeltos, y con muros delgados, de espesores entre 15 y $20 \mathrm{~cm}$ (Massone et al., 2012).

Se debe hacer énfasis que el buen comportamiento observado en general en edificios en Chile en el terremoto de 1985, llevó posteriormente a la práctica mencionada de edificios más altos con menores espesores de muros. Además, se debe reconocer la ventaja de un edificio con muros estructurales respecto a uno con marcos, ya que los primeros evitan modos de falla indeseables como pueden ser el que los pisos colapsen uno encima de otro, como se observó en la ciudad de México en edificios con marcos, particularmente aquellos con losas reticulares sin vigas. Este buen comportamiento de edificios en Chile en el terremoto de 1985 se debió al empleo en edificios de CR de espesores de muros generosos, del orden de $20 \mathrm{~cm}$ a $30 \mathrm{~cm}$, siendo esta última dimensión la más común antes de 1985 (Massone et al., 2012), así como a la buena calidad del suelo en la mayor parte de las áreas afectadas por el terremoto (Bonelli, 2015).

Un parámetro importante que posteriormente se utiliza en esta investigación para la evaluación del comportamiento sísmico de edificios de CR es la relación $H / T_{o}$, donde $H$ es la altura del edificio en metros, y $T_{o}$ es el periodo fundamental del edificio en la condición de secciones no agrietadas. Las estadísticas de esta relación para edificios construidos en Chile en el periodo 1940 a 2010 muestra valores en un intervalo amplio, entre $40 \mathrm{~m} / \mathrm{s}$ y $150 \mathrm{~m} / \mathrm{s}$, con una media de $70 \mathrm{~m} / \mathrm{s}$ (Massone et al., 2012; Lagos et al., 2012).

Para el estudio de este terremoto se eligió la ciudad de Concepción, donde ocurrieron los mayores daños en edificios en este evento. En edificios de más de 10 niveles en Concepción, en alrededor del 10\% de estos edificios se observó daño estructural (Massone et al, 2012). Se seleccionaron los registros de aceleraciones de la estación Concepción Centro, el estudio de los registros de esta estación es relevante porque se ubica en la zona de la ciudad donde se concentraron los daños en edificios. Los registros empleados de la estación Concepción Centro son las componentes horizontales $L$ y $T$. Algunas de las características de los registros de esta estación se muestran en la Tabla 1. Para la interpretación de la respuesta de estructuras en esta estación es importante considerar el tipo de suelo en la estación. La estación Concepción Centro se ubica en un suelo arenoso, y por la velocidad de onda de corte en el lugar, ver Tabla 1, $V_{s}$, se clasificaría como tipo D según la clasificación de la ASCE 7-10 (ASCE 7-10, 2010).

Las figuras 1c y 1d muestran los espectros de aceleraciones y desplazamientos elásticos para los registros seleccionados de la estación Concepción Centro, en sus dos componentes horizontales, y fueron calculados con un porcentaje del amortiguamiento crítico igual a 5\%. También las figuras 1c y 1d muestran los espectros de diseño elásticos especificados por la normativa de diseño sísmico vigente en la fecha del terremoto, NCh 433 (NCh 4333, 1996), para los casos de suelos clasificados por esa normativa como suelos tipos III y IV. La zona de mayor daño estructural en Concepción se clasificaba como suelo tipo III, varios edificios dañados en esa ciudad tenían muros delgados y fueron diseñados para un suelo tipo II (Bonelli, 2015). Los resultados de las Figs 1c y 1d muestran que las ordenadas espectrales elásticas calculadas para registros de la estación Concepción Centro, en sus componentes horizontales L y T, son bastante mayores que las especificadas por la normativa local para suelos Tipo III, en la zona de periodos estructurales, $T$, en el intervalo aproximado de $1.5 \mathrm{~s}$ a $3 \mathrm{~s}$. Las figuras $1 \mathrm{c}$ y $1 \mathrm{~d}$ muestran que el intervalo de periodos entre alrededor de $1.5 \mathrm{~s}$ a $2.5 \mathrm{~s}$, las ordenadas espectrales de los registros de la Estación concepción Centro superaron incluso a los valores especificados por la normativa NCH 433 para estructuras en suelo Tipo IV. El tipo de suelo IV corresponde a suelos cohesivos saturados con valores de $s_{u}$ entre 0.025 y $0.1 \mathrm{MPa}$, donde $s_{u}$ es la resistencia al corte no drenada del suelo (NCh 433, 1996). Estos 
resultados indican que, como en el caso del análisis de los terremotos de Christchurch en 2010 y 2011 , las ordenadas espectrales elásticas calculadas con registros de aceleraciones del lugar superaron ampliamente a las ordenadas especificadas por la normativa vigente.

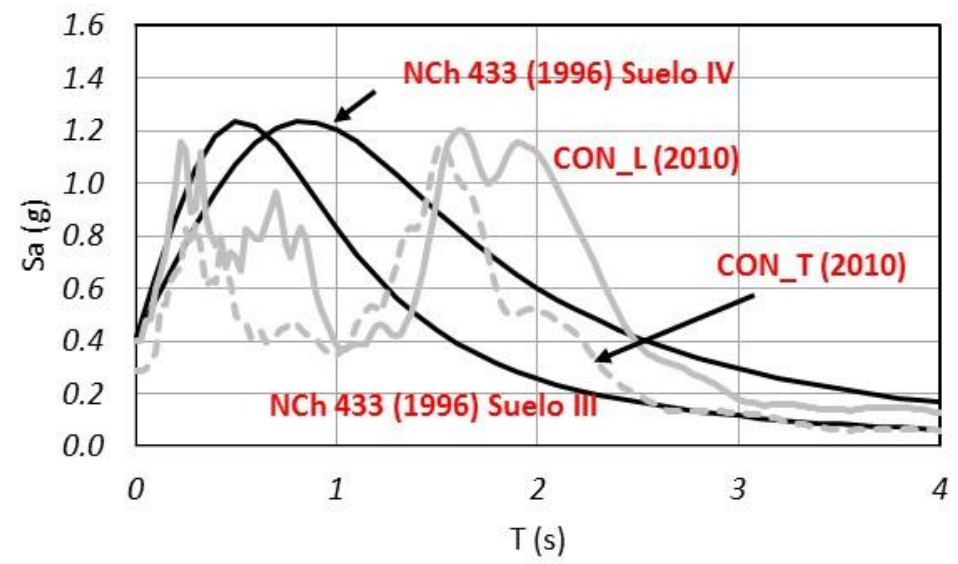

c) Aceleraciones espectrales horizontales obtenidas con registros de la estación Concepción Centro en el terremoto de Maule, Chile 2011

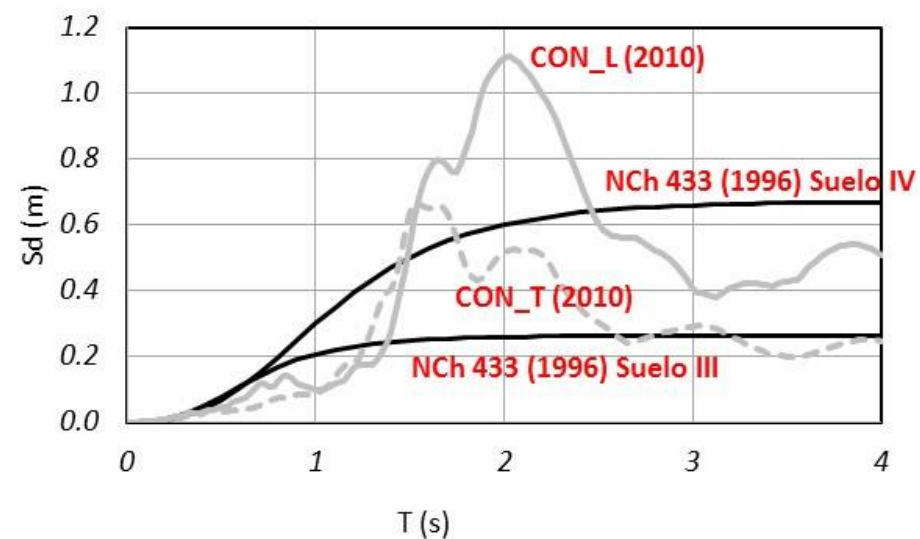

d) Desplazamientos espectrales horizontales obtenidos con registros de la estación Concepción Centro en el terremoto de Maule, Chile 2011

Figura 1. Aceleraciones y desplazamientos espectrales horizontales obtenidos con registros de aceleraciones de los terremotos de México 1985; Maule, Chile 2011, y Christchurch 2010 y 2011 (Continuación)

\section{El terremoto del 19 de Septiembre 1985 en la Ciudad de México}

El terremoto del 19 de Septiembre de 1985 con epicentro en la costa de Michoacán, causó colapsos o daños severos en cientos de edificios en la ciudad de México, principalmente en edificios con marcos de concreto reforzado, con mayor incidencia en edificios con losas reticulares sin vigas. Este evento causó la pérdida de miles de vidas humanas. Su estudio es importante porque en la zona de mayores daños se obtuvo el registro de aceleraciones de la estación SCT, ver Tabla 1, lo que permite obtener espectros de respuesta lineal e inelásticos, así como resultados de análisis dinámicos inelásticos de estructuras, con el fin de compararlos con el comportamiento observado de edificios en este terremoto. Sin 
embargo, es posible que en ciertas áreas de la zona de daños las demandas de desplazamientos hayan sido mayores que las correspondientes a la estación SCT, por lo tanto los resultados del estudio de estructuras empleando este registro no se pueden considerar conservadores. Las figuras 1e y 1f muestran espectros de aceleraciones y desplazamientos elásticos para el registro de aceleraciones obtenido en la estación SCT para la componente horizontal $\mathrm{EW}$, y fueron calculados con un porcentaje del amortiguamiento crítico igual a 5\%. Las figuras 1e y 1f también muestran los espectros de aceleraciones y desplazamientos obtenidos con las especificaciones de la normativa vigente en la fecha del terremoto, la cual fue el Reglamento de Construcciones para el Distrito Federal de 1976 (RCDF, 1976), para lo cual las ordenadas espectrales especificadas se multiplicaron por el factor de sobre resistencia de estructuras, el cual en este estudio se consideró igual a 2. Se aprecia que en zona de periodos entre aproximadamente $1.5 \mathrm{~s}$ a $2.8 \mathrm{~s}$, las ordenadas espectrales elásticas calculadas para el registro SCT en su componente EW, son mayores que las que se obtendrían con las especificaciones del RCDF 1976, con la mayor diferencia para $T$ alrededor de 2 s.

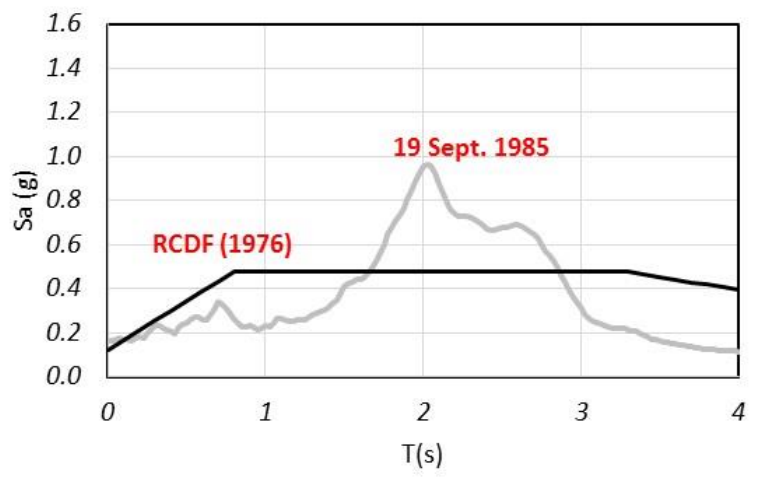

e) Aceleraciones espectrales obtenidas con el registro SCT-EW en el terremoto de México 1985

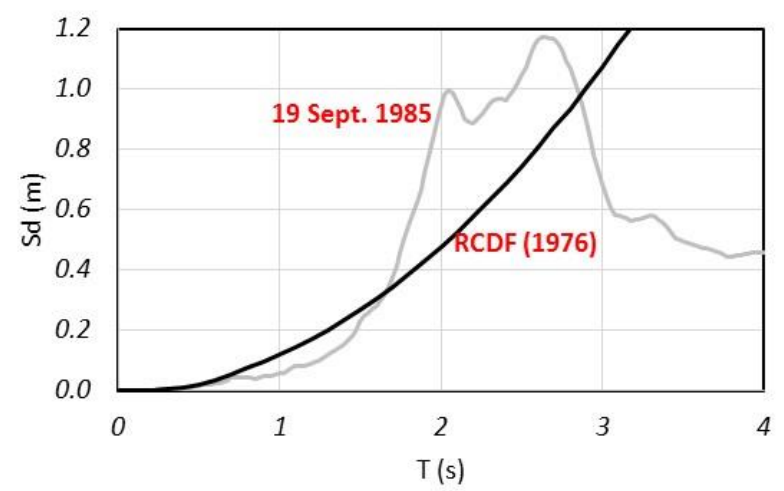

f) Desplazamientos espectrales obtenidos con el registro SCT-EW en el terremoto de México 1985

Figura 1. Aceleraciones y desplazamientos espectrales obtenidos con registros de aceleraciones de los terremotos de México 1985; Maule, Chile 2011, y Christchurch 2010 y 2011 (Continuación)

Noreña et al. (1989) muestran resultados de estadísticas de colapsos o daños en edificaciones existentes en el evento en estudio en la Delegación Cuauhtémoc. El total de edificaciones de uno o más niveles en esta Delegación fue igual a 44,350. El número total de edificaciones en esta área de la ciudad con colapsos o daños severos fue 1,397, lo que representa el 3.1\% del total de edificaciones existentes en esta Delegación o el 61\% del número de edificaciones dañadas en toda la ciudad (Noreña et al., 1989). Sin embargo, una evaluación de la distribución de daños en función del número de niveles de las edificaciones muestra resultados que merecen tomarse en cuenta para la interpretación de estos daños. Como muestra la 
figura 2, se encontró que la incidencia de daños más alta fue en edificios de más de ocho niveles. El número total de edificios de más de ocho niveles fue 637, de esta población 232 edificios mostraron colapsos o daños severos, lo que representa el 36\% del número de edificios. Esta incidencia de daños o colapsos fue bastante mayor que el observado en intervalos de menor número de niveles. Por ejemplo, del número de edificaciones existentes de 1 a 5 niveles, el 2.3\% llegaron al colapso o mostraron daños severos, y del número de edificaciones existentes de 6 a 8 niveles el porcentaje de daños o colapsos fue $14.3 \%$ (Noreña et al., 1989).

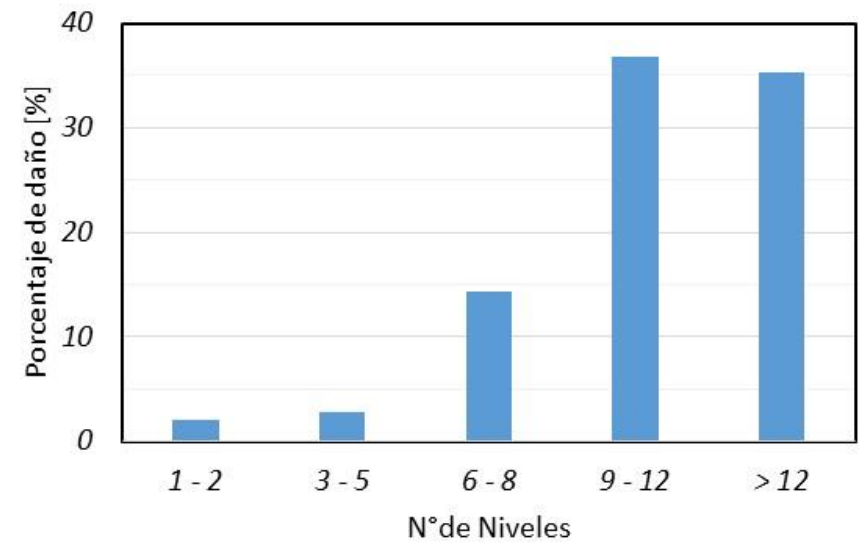

Figura 2. Distribución observada de colapsos o daños severos en edificios en la ciudad de México en el terremoto de 1985 (Noreña et al., 1989)

\section{Comentarios a los resultados obtenidos para los terremotos estudiados}

Los valores de las demandas de aceleraciones y desplazamientos elásticos, calculados con registros de aceleraciones en los terremotos que aquí se estudian, en todos los casos fueron mayores que los especificados por normativas de los diversos lugares que experimentaron estos terremotos, con valores que en algunos casos excedieron a los especificados en alrededor de $100 \%$. En los terremotos estudiados se observaron diversos niveles de daños o colapsos en edificaciones, que no eran esperados por los ingenieros que diseñaron estas edificaciones. Esto muestra incertidumbres considerables en los procedimientos de diseño sísmico especificados por normativas de construcción. Además, los resultados mostrados sugieren que las hipótesis empleadas para definir las acciones sísmicas de diseño merecen ser revisadas, y como muestra Gerstenberger et al. (2014) es posible que se requieran nuevos procedimientos para estimar las ordenadas espectrales de diseño.

En la práctica de diseño sísmico se acepta que es antieconómico el diseñar un sistema estructural tradicional con respuesta elástica, ya que como fue el caso de los terremotos de Christchurch en 2010 y 2011, la estructura durante su vida útil en un terremoto alcanzará su resistencia lateral, incluso en tiempos muchos menores que el de la vida útil como en el caso de los referidos terremotos. Se infieren dos observaciones. La primera es que debido a que en un terremoto la estructura alcanzará su resistencia lateral, para esta resistencia se debe llevar a cabo un diseño por capacidad; es decir, evitar modos de falla indeseables. Estos son por ejemplo modos de falla por cortante, agotamiento prematuro de la capacidad de deformación de secciones críticas de elementos estructurales, punzonamiento en el caso de edificios con losas reticulares sin vigas, entre otros. La segunda observación es que además de considerar la resistencia lateral de una estructura de acuerdo con normativas de construcción, es necesario diseñar la estructura con capacidad de desplazamientos o distorsiones por sismo que sea mayor que la demanda esperada en el terremoto de diseño. Además, es conveniente emplear un sistema estructural sismo 
resistente que tenga la característica de controlar esta demanda, como es el sistema con muros estructurales. Los daños severos o colapsos observados en edificios en la ciudad de México en 1985 indican que en ese momento el estado del arte en el diseño sísmico era el hacer énfasis en la resistencia lateral de la estructura, y menos énfasis en la revisión de las distorsiones en la estructura, así como para considerar las posibles incursiones inelásticas de las estructuras en terremotos y el detallado del refuerzo necesario para estas incursiones. Esto se elabora en lo siguiente.

\section{ANÁLISIS APROXIMADO DE LA RESPUESTA INELÁSTICA DE ESTRUCTURAS EN LOS TERREMOTOS DE 1985 MEXICO Y 2010 CHILE}

Es de interés comparar el comportamiento observado de estructuras en la ciudad de México durante el terremoto de 1985 con el observado en Concepción en el terremoto de Maule de 2010. Para este fin se consideró conveniente analizar resultados de espectros de respuesta tanto elásticos como inelásticos de registros típicos en la zona de daños en ambos terremotos. Para el caso de la ciudad de México se seleccionó el registro de aceleraciones SCT-EW. Para el caso de Concepción se seleccionó el registro de la estación Concepción Centro, CON-L, así como el registro CCSP EW de la estación Concepción San Pedro.

Los espectros de respuesta fueron obtenidos con el programa Ruaumoko (Carr, 2011), el cual permite evaluar la respuesta dinámica no lineal de una estructura, en este caso de 1 grado de libertad (1GDL). El paso de integración empleado fue igual a 0.001. Para representar el comportamiento inelástico de una estructura de concreto reforzado se empleó la regla de histéresis de Takeda modificado (Carr, 2011), en la que la rigidez de descarga, $k_{u}$, se definió con el parámetro $\alpha$ igual a 0.4 y el tipo de descarga empleado fue el denominado Drain-2D por el programa Ruaumoko. Para la rigidez de carga después de la fluencia se empleó el parámetro $\beta$, definido en el referido programa, igual a 0.6. Los valores seleccionados de los parámetros $\alpha$ y $\beta$ están dentro del intervalo de valores sugeridos para el empleo de la regla de histéresis de Takeda modificado (Carr, 2011). La fracción de amortiguamiento crítico, $\xi$, que se empleó fue igual a $5 \%$, con el fin de ser congruente con los valores de la fracción de amortiguamiento crítico implícito en los espectros de diseño de las normativas. Sin embargo, se debe mencionar que este valor para un análisis dinámico inelástico podría estar sobrestimado, ya que resultados de ensayes en mesa vibradora de un edificio de concreto reforzado de 7 niveles (Panagiotou, 2008; Martinelli y Filippou, 2009 ) indican que cuando se lleven a cabo análisis dinámicos no lineales, emplear valores de $\xi$ mayores que $2 \%$ lleva a subestimar de manera importante los desplazamientos laterales en la estructura. Por esta razón, resultados de análisis dinámicos inelásticos con un valor de $\xi$ igual a $5 \%$, no se podrían considerar conservadores.

\section{Espectros de respuesta para el registro SCT}

Las figuras $3 \mathrm{a}$ y $3 \mathrm{~b}$ muestran resultados de espectros de aceleraciones y desplazamientos, $S_{a}$ y $S_{d}$, respectivamente, obtenidos con el registro SCT-EW, tanto para respuesta elástica como inelástica. La respuesta inelástica se hace función de la ductilidad de desplazamiento, $\mu$, de la estructura, para la cual se emplean los valores de 2 y 4 , casos que en estas figuras se denominan DUC $=2$ _TAK y DUC $=4$ _TAK, respectivamente. El caso elástico se denomina $\mathrm{DUC}=1$.

En el caso de la respuesta espectral de desplazamientos, $S_{d}$, es de interés observar que a diferencia de espectros inelásticos en terreno firme, en el caso del registro SCT no se cumple la regla de "igual desplazamientos" (desplazamientos elásticos e inelásticos son iguales), característica que comúnmente se atribuye a suelos blandos de arcilla como el de la ciudad de México, pero que como se muestra posteriormente también se observa en suelos diferentes. Se aprecia además, que en el intervalo de periodos 
de $1 \mathrm{~s}$ a $1.5 \mathrm{~s}$, los desplazamientos espectrales para el caso $\mu=4$ son bastante mayores que los del caso $\mu=2$, situación que cambia para periodos mayores que $1.7 \mathrm{~s}$, figura $3 \mathrm{~b}$. Esto muestra que cuando no se cumple la regla de igual desplazamientos, como es el caso que se analiza, un procedimiento para reducir la demanda de desplazamientos, dependería no sólo de la ductilidad que se emplee, sino también del intervalo de periodos de interés.

Se debe mencionar que para el espectro de respuesta de desplazamientos para el caso $\mu=4$, en el intervalo de periodos entre $0.95 \mathrm{~s}$ y $1.45 \mathrm{~s}$, el procedimiento del programa Ruaumoko logró la convergencia con valores algo mayores que la ductilidad objetivo de 4 , excediendo este valor entre $5 \%$ y $15 \%$, en este intervalo de periodos.

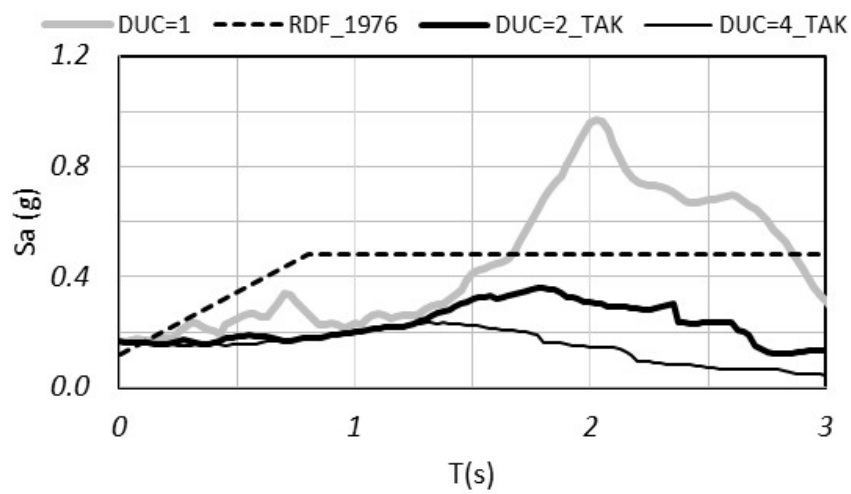

(a) Aceleraciones espectrales horizontales obtenidas para la estación SCT en el terremoto de México 1985, y espectro de diseño

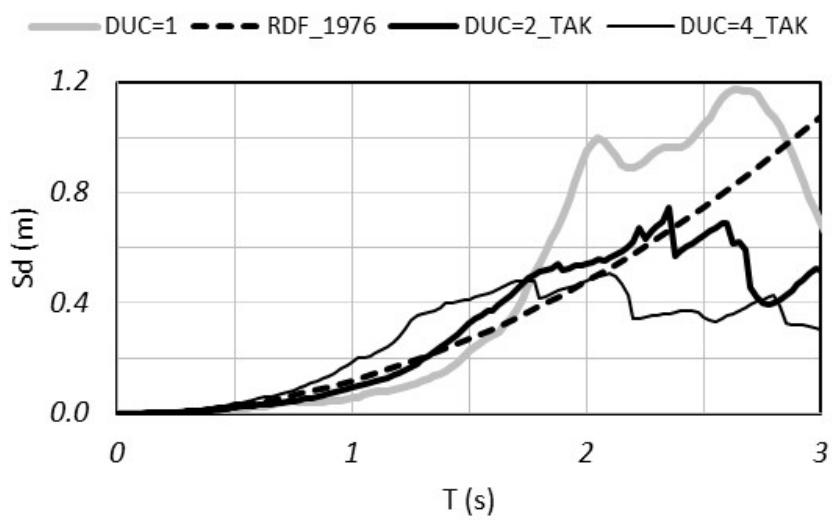

(b) Desplazamientos espectrales horizontales para la estación SCT en el terremoto de México 1985, y espectro de diseño

Figura 3. Aceleraciones y desplazamientos espectrales horizontales obtenidos para la estación SCT en el terremoto de México 1985, y espectro de diseño

\section{Espectros de respuesta para registros en Concepción}

Las figuras $4 \mathrm{a}$ y $4 \mathrm{~b}$ muestran resultados de espectros de aceleraciones y desplazamientos, $S_{a}$ y $S_{d}$, respectivamente, empleando el registro $\mathrm{CON}-\mathrm{L}$, obtenido en la zona de mayor daño estructural en Concepción. Una comparativa entre los espectros para el caso de la ciudad de México, figura 3, y los de Concepción Centro no muestra grandes diferencias en estos dos casos, por ejemplo, en ambos casos no se 
cumple la regla de "igual desplazamientos", además también en ambos casos, la variación de los desplazamientos espectrales en función de la ductilidad dependen del intervalo de periodos. Es de interés observar que las aceleraciones espectrales tanto elásticas como inelásticas para el registro CON-L en Concepción son incluso mayores que las correspondientes al registro SCT en un intervalo amplio de periodos (periodos menores que alrededor de 2s), figuras 3a y 4a. Una tendencia semejante se observa para el caso de espectros de desplazamientos, figuras $3 \mathrm{~b}$ y $4 \mathrm{~b}$. Con estos resultados se podría pensar que era de esperar mayores daños o colapsos en Concepción que en el caso de la ciudad de México, lo cual no fue así como se ha comentado antes. Posteriormente se postula una explicación para este comportamiento de edificios en ambos eventos.

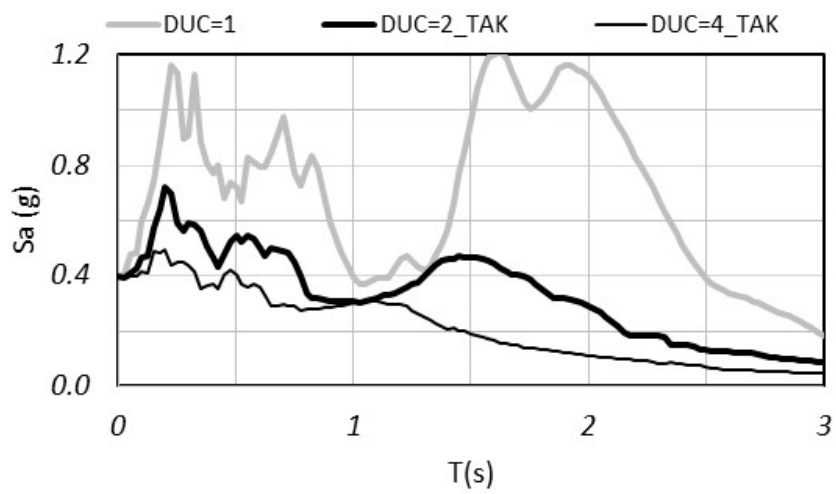

(a) Aceleraciones espectrales horizontales obtenidas para la estación Concepción Centro en el terremoto de Maule, Chile 2010

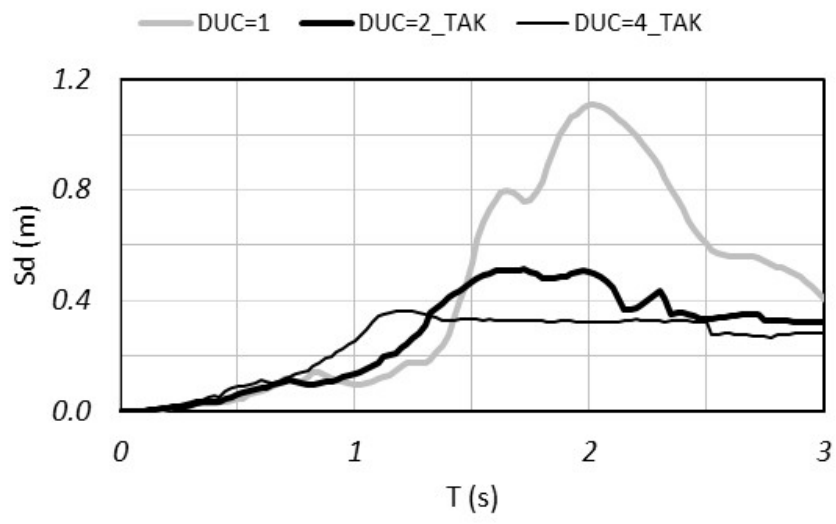

(b) Desplazamientos espectrales horizontales obtenidos para la estación Concepción Centro en el terremoto de Maule, Chile 2010

Figura 4. Aceleraciones y desplazamientos espectrales horizontales obtenidos para la estación Concepción Centro en el terremoto de Maule, Chile 2010

El caso del análisis del registro de la estación Concepción San Pedro, CCSP EW, es de interés como se muestra en lo siguiente. Esta estación está ubicada en una zona de la ciudad a $5 \mathrm{~km}$ de la estación Concepción Centro, zona en la que no se observó daños importantes en estructuras. Algunas de las características de los registros de esta estación se muestran en la tabla 1. La estación Concepción San Pedro se ubica en un suelo de grava y arena, y se clasificaría como suelo tipo C (Boroschek et al., 2012), si se considera el valor en el sitio de la velocidad de onda de corte que fue igual a $390 \mathrm{~m} / \mathrm{s}$, ver tabla 1 . 
Las figuras 5 a y 5 b muestran resultados de espectros de aceleraciones y desplazamientos, $S_{a}$ y $S_{d}$, respectivamente, obtenidos con el registro CCSP EW para las respuestas elásticas e inelásticas. Se observa que en general las ordenadas espectrales para este registro son menores que las mostradas para la estación Concepción Centro. Además, las características de los espectros con el registro CCSP EW son muy diferentes a los de la estación Concepción Centro, con una respuesta típica de estructuras en suelo firme. Por ejemplo, las reducciones de resistencia respecto a las demandas elásticas son aproximadamente proporcionales a los valores de $\mu$ en un intervalo amplio de periodos, así como que en general se cumple la regla de "igual desplazamientos". Estos resultados muestran la relevancia del tipo de suelo en la respuesta sísmica de una estructura, así como los cambios importantes de demandas sísmicas en distancias relativamente pequeñas en una ciudad. Es de interés mencionar que considerando estas características observadas en el terremoto de Maule 2010, en la nueva normativa chilena para el diseño sísmico de edificios se ha ampliado el número de tipos de suelos a considerar en el diseño sísmico, pasando de cuatro, que se contaba antes del terremoto de 2010, a seis (Guendelman et al., 2012).

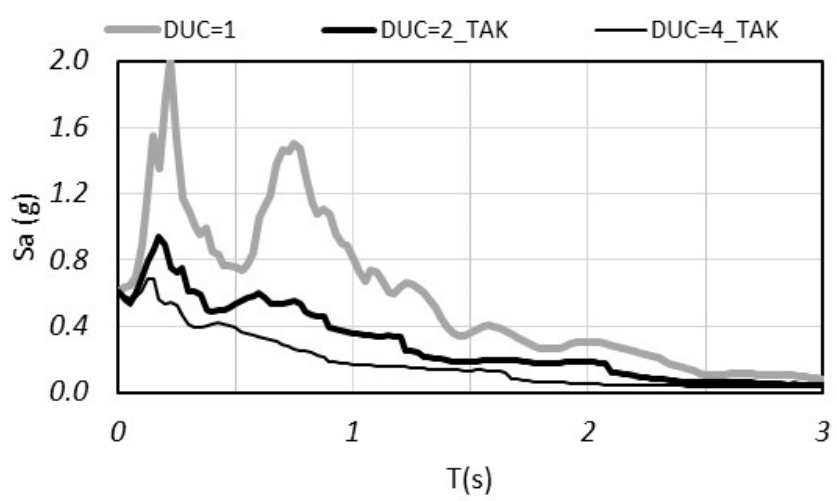

(a) Aceleraciones espectrales horizontales obtenidas en la estación Concepción San Pedro en el terremoto de Maule, Chile 2010

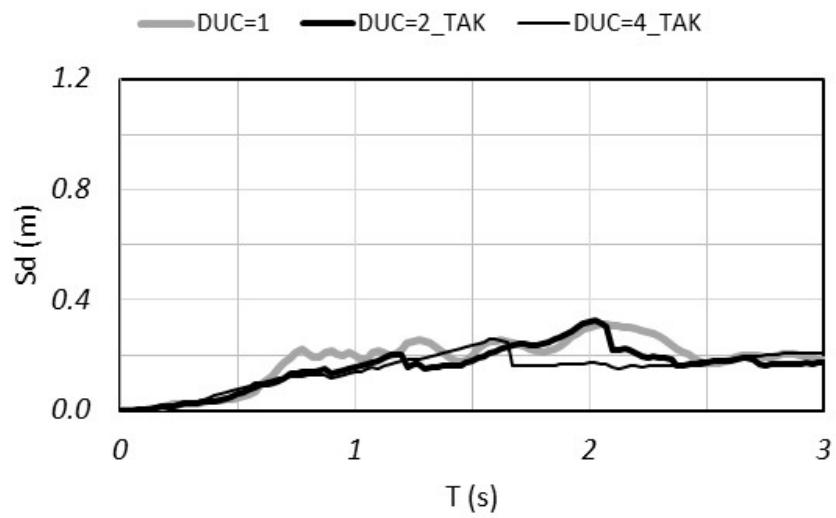

(b) Desplazamientos espectrales horizontales obtenidos en la estación Concepción San Pedro en el terremoto de Maule, Chile 2010

Figura 5. Aceleraciones y desplazamientos espectrales horizontales obtenidos en la estación Concepción San Pedro en el terremoto de Maule, Chile 2010 


\section{Análisis aproximado de desplazamientos laterales en edificios para los casos de los terremotos estudiados}

El análisis de ordenadas espectrales elásticas é inelásticas, no es suficiente para una interpretación razonable del comportamiento sísmico estructuras, principalmente debido a que estas ordenadas no consideran el tipo de estructuración de un edificio, como se muestra en lo que sigue.

Para el análisis aproximado de la demanda de desplazamientos en un edificio, conociendo las ordenadas espectrales, se emplea el conocido concepto de la estructura equivalente, la cual es una estructura de 1GDL con el mismo periodo y ductilidad de desplazamiento que tendría el edificio con varios grados de libertad (Saiidi y Sozen, 1981). En lo que sigue se hace un resumen del este procedimiento.

La distorsión global máxima de un edificio, $D_{r m}$, se define como:

$$
D_{r m}=\frac{\delta_{m}}{H}
$$

donde $\delta_{m}$ es el desplazamiento lateral máximo del último nivel del edificio sometido a un movimiento del terreno, y $H$ la altura del edificio. Se emplea la hipótesis de que $\delta_{m}$ se puede calcular multiplicando el desplazamiento espectral, $S_{d}$, por el factor de participación $\Gamma$, el valor sugerido para este parámetro para un análisis aproximado de desplazamientos en edificios regulares es 5/4 (Sozen, 1997). El autor considera que la simplicidad es relevante cuando se desea estudiar problemas difíciles, como es en este caso la selección de un valor que se considere adecuado para $\Gamma$, cuando se emplee para el cómputo de distorsiones globales en una población amplia de edificios. Una estimación precisa de $\Gamma$ depende del sistema estructural (así como del número de niveles del edificio en estudio). En Ghosh (2016), se muestran valores de $\Gamma$ que se emplearán en la normativa ASCE 7-16 (2017) para el diseño sísmico de diafragmas de edificios. De acuerdo con Ghosh (2016) los valores de $\Gamma$ son mayores en los casos de edificios con muros respecto a los de edificios a base de marcos. Sin embargo, considerando las altas incertidumbres que intervienen en el problema de la predicción del comportamiento sísmico de estructuras, en opinión del autor las diferencias entre los probables valores de $\Gamma$ para edificios a base de muros y a base de marcos no serían significativas, y no justifican el empleo de un valor diferente de $\Gamma$ para un análisis sísmico simplificado como el empleado en esta investigación.

Una expresión aproximada sencilla que se emplea para estimar el periodo fundamental, $T$, de un edificio es:

$T=\frac{n}{\lambda}$

donde $n$ es el número de niveles del edificio y $\lambda$ es un factor que depende del tipo de sistema estructural del edificio, y tiene unidades $s^{-1}$. Con el empleo de la ecuación 4, la altura $H$ en un edificio regular con altura de entrepiso, $h$, constante, se puede expresar como:

$$
H=T \lambda h
$$

De las euaciones 3 a 5 y del valor aproximado propuesto para definir $\delta_{m}$ se obtiene: 


$$
D_{r m}=\frac{\Gamma S_{d}}{T \lambda h}
$$

Como muestra la ecuación 6 , en el cómputo de $D_{r m}$ se emplea el producto $\lambda h$, como indica la ecuación 5 este producto se puede reemplazar por la relación $H / T$. Como se ha mencionado, para el caso de edificios en Chile existen valores medidos de la relación $H / T_{o}$, la cual como se muestra más adelante se puede considerar igual a $\sqrt{2} H / T$. Cuando se conozcan, como en el caso de edificios en Chile, valores de la relación $H / T$, es conveniente emplear esta relación en el cálculo de $D_{r m}$. De las ecuaciones 5 y 6 se tiene:

$$
D_{r m}=\frac{\Gamma S_{d}}{T(H / T)}
$$

La ecuación 7 muestra la importancia de la relación $H / T$ para disminuir la demanda de distorsiones en un edificio durante un terremoto. Esta relación se emplea también en la definición de un índice de daño que se comenta posteriormente. En el caso de estructuras con marcos para una estimación del periodo inicial del edificio con secciones sin agrietar, es común emplear para $\lambda$ en la ec (4) el valor $10 s^{-1}$. Si se considera la hipótesis de que la rigidez efectiva de edificios con secciones agrietadas es del orden de la mitad de la rigidez lateral considerada con secciones sin agrietar (Shimazaki and Sozen, 1984), el periodo efectivo, $T$, sería $\sqrt{ } 2$ veces el periodo inicial $T_{o}$. Con esta hipótesis y considerando además la amplificación del periodo por la interacción suelo-estructura se tiene que $\lambda$ es igual a $10 /(\sqrt{ } 2 * 1.3)=5.4 s^{-1}$ (Rodriguez y Aristizabal, 1999). Como en el caso aquí comentado de la selección de un valor representativo de $\Gamma$, también la simplicidad es relevante para la selección de valores representativos para $\lambda$. Otros autores (Elwood y Eberhard, 2009) han mostrado que las normativas sobrestiman de manera importante la rigidez lateral efectiva de estructuras, lo que llevaría a periodos efectivos en la estructura mayores que los supuestos tradicionalmente de acuerdo con normativas. Esto implica que para la estimación de periodos efectivos es razonable el empleo de una estimación que además de simplista sea razonablemente conservadora, como el autor considera se está haciendo en esta investigación.

Para el caso del análisis aproximado de edificios a base de muros estructurales de CR, típicos de la práctica chilena, Rodriguez y Aristizabal (1999) propusieron para $\lambda$ el valor $14 \mathrm{~s}^{-1}$. Es conveniente revisar si este valor de $\lambda$ podría ser razonable considerando información reciente. El valor promedio de $H / T_{o}$ medido en edificios chilenos fue igual a $70 \mathrm{~m} / \mathrm{s}$ (Massone et al., 2012). Considerando la expresión $T=\sqrt{ } 2$ $T_{o}$, , esta relación de $H / T_{o}$ se convierte en la relación $H / T=50 \mathrm{~m} / \mathrm{s}$. Si en la ecuación 5 empleamos para $h$ el valor $3 m$, y para $\lambda$ el valor $14 s^{-1}$ se obtendría el valor $42 \mathrm{~m} / \mathrm{s}$ para $H / T$, valor no muy lejano del referido valor promedio observado de $50 \mathrm{~m} / \mathrm{s}$, lo que se puede considerar aceptable dada la variabilidad medida para esta relación.

Para el cómputo de $D_{r m}$ con la ecuación 6, los valores empleados para $S_{d}$ fueron los obtenidos de los espectros de desplazamientos, $S_{d}$, anteriormente descritos. Además, se emplearon para edificios en México y Chile los valores $5 / 4$ y $3 m$, para $\Gamma$ y $h$, respectivamente. Como se ha mencionado, para $\lambda$ se emplearon los valores $5.4 s^{-1}$ y $14 s^{-1}$ para edificios con marcos en la ciudad de México y edificios con muros en Chile, respectivamente. Con los espectros de desplazamientos para los registros SCT y CON-L, de los terremotos de Mexico 1985 y Chile 2010, se obtuvieron los resultados mostrados en la figura 6. Estos resultados corresponden además del caso de respuesta elástica, los casos de valores para $\mu$ iguales a 2 y 4. A pesar de que como se ha comentado las diferencias de los valores de $S_{d}$ en estos eventos no fueron importantes, los resultados mostrados en la figura 6 indican claramente la importante diferencia de distorsiones globales máximas obtenidas para edificios típicos en los casos de los terremotos de Mexico 1985 y Chile 2010, en lugares representativos de estos eventos. 


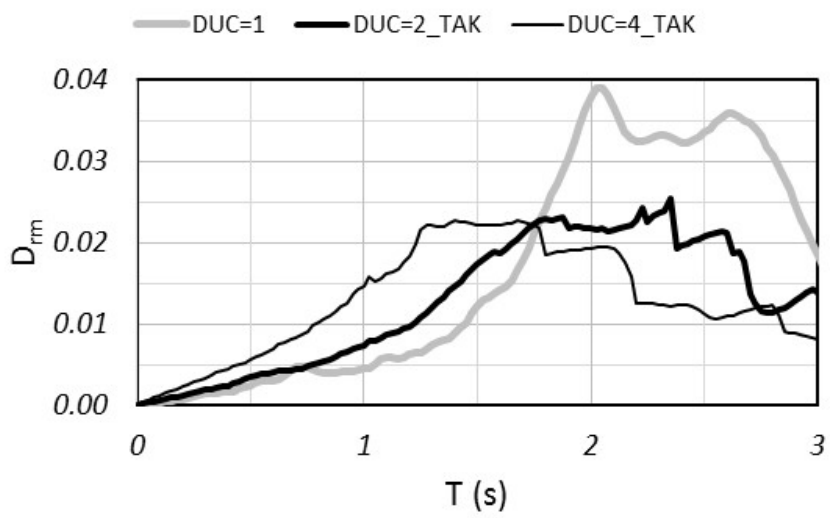

(a) Valores de $D_{r m}$ obtenidos con el registro SCT en el terremoto de México 1985.

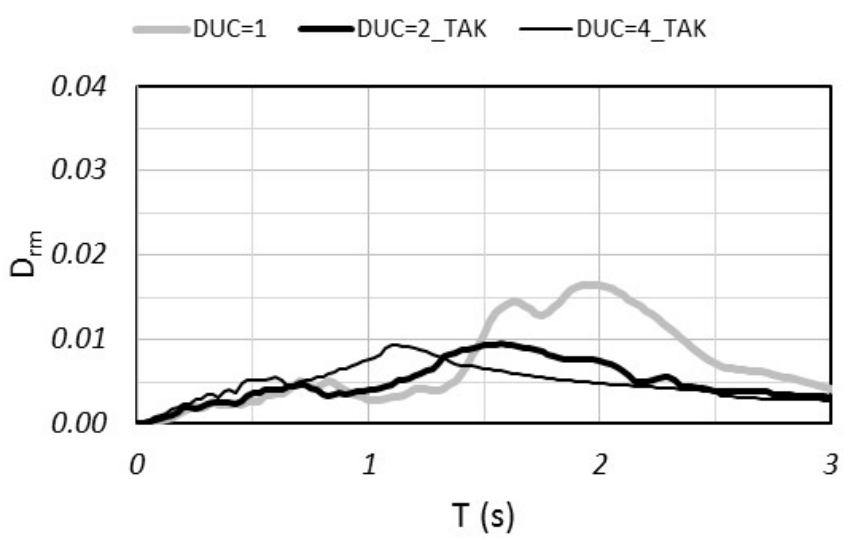

(b) Valores de $D_{r m}$ obtenidos con el registro CON-L en la estación Concepción Centro en el terremoto de Maule, Chile 2010.

Figura 6. Distorsiones globales en edificios, $D_{r m}$, obtenidas con registros de estaciones en los terremotos de México 1985, y Maule, Chile 2010

De la figura 6 se puede inferir dos conclusiones relevantes que ayudan a explicar la mayor incidencia observada de daños así como colapsos de edificios, y muertes, en la ciudad de México en 1985, respecto a lo observado en Concepción en 2010. La primera conclusión es que debido principalmente a la alta flexibilidad inherente a sistemas estructurales con marcos, en estos terremotos las distorsiones globales de edificios típicos en la ciudad de México, en un intervalo amplio de periodos estructurales, habrían sido del orden del doble de los que experimentaron edificios en Concepción con periodos estructurales semejantes. La segunda conclusión es que los valores de distorsiones globales computadas para los edificios de la ciudad de México en un intervalo amplio de periodos son bastante mayores que 0.01, valor considerado como límite para un aceptable comportamiento ante terremotos de edificios con marcos o muros (Rodriguez y Aristizabal, 1999), mientras que en el caso de edificios en Concepción los resultados muestran que las distorsiones globales en estos edificios fueron menores que el referido valor límite, aunque se acercaron a este valor en un intervalo pequeño de periodos, de alrededor de $1.1 \mathrm{~s}$ a $1.6 \mathrm{~s}$, figura 6. El único caso de colapso observado en Concepción fue el de un edificios de 15 niveles construido en 2007 (Wallace et al., 2012). Estas conclusiones son también congruentes con resultados encontrados con un índice de daño aplicado a edificios típicos en estos eventos que se describen en la siguiente sección. 


\section{Empleo de un índice de daño para el análisis de la respuesta de edificios en los terremotos de 1985 en la ciudad de México y en Concepción en 2010}

Rodriguez y Aristizabal (1999) propusieron un parámetro de daño por sismo en estructuras empleando registros de aceleraciones del terreno obtenidos en terremotos típicos en el mundo en esa época. Los resultados encontrados para este parámetro ordenaron de manera aceptable el daño observado en esos terremotos. Rodriguez (2015) convirtió este parámetro en un índice de daño, donde 0 indica no daño y 1 indica colapso. Para el cómputo de este índice también se emplean registros de aceleraciones del terreno, y los terremotos considerados fueron, además de la mayoría empleados por Rodriguez y Aristizabal (1999), los terremotos de Chile 2010, Japón 2011 y Christchurch, Nueva Zelanda en 2011. Los resultados encontrados con este índice de daño fueron congruentes con los daños observados en los terremotos considerados (Rodriguez, 2015). En lo que sigue se describe de manera breve este índice de daño y se muestra resultados encontrados con este índice para los casos de edificios típicos en los terremotos en la ciudad de México en 1985 y en Concepción en Chile en 2010.

El índice de daño, $I_{d}$, propuesto por Rodriguez (2015), se basa en el empleo de la misma estructura de 1GDL descrita para el cómputo aproximado de $D_{r m}$ aquí descrito, y se define como:

$$
I_{d}=\frac{\Gamma^{2} E_{H}}{\left(\omega H D_{r c}\right)^{2}}
$$

donde $E_{H}$ es la energía histerética, definida como el trabajo inelástico de una estructura de 1GDL cuando se analiza con un registro de aceleraciones. El parámetro $D_{r c}$ es un valor seleccionado para una distorsión global de referencia en un edificio y es igual a 0.025 (Rodriguez, 2015). Este valor de $D_{r c}$ aplica a edificios diseñados con reglamentos modernos sismo resistentes (Rodriguez, 2015). Esto lleva a que para casos de diseño en México con $Q=2$, el referido valor de $D_{r c}$ estaría sobrestimado, lo que llevaría a resultados del lado de la inseguridad en el cómputo de $I_{d}$. El parámetro $\omega$ es la frecuencia circular de la estructura de $1 \mathrm{GDL}$, igual a $2 \pi / T$, donde $T$ es el periodo fundamental del edificio. Con la definición de $\omega$, la ecuación 8 se convierte en

$$
I_{d}=\frac{\Gamma^{2} E_{H}}{\left(2 \pi \frac{H}{T} D_{r c}\right)^{2}}
$$

La ventaja de la ecuación 9 respecto a la ecuación 8 es que introduce la relación $H / T$ aquí comentada, y muestra la relevancia de esta relación para disminuir el daño esperado en edificios en terremotos. Lagos et al. (2012) han empleado la relación $H / T$ en su versión $H / T_{o}$, para la evaluación del comportamiento sísmico de edificios en el terremoto de Maule, Chile 2010, y han mostrado que el aumento del valor de este parámetro, mediante el empleo de estructuras rígidas, se relacionó con menores niveles de desplazamientos laterales y daños en edificios en este terremoto. Estas observaciones son congruentes con la ecuación 9, la que indica que estructuras rígidas tendrían menor daño por sismo respecto a estructuras flexibles.

La ecuación 9 indica que para un sistema estructural dado, con un valor constante de $H / T$, el índice de daño $I_{d}$ en función del periodo, es el producto de una constante por los valores de $E_{H}$ en función del periodo. Esta energía es una demanda, y lleva en este caso a que la forma de la distribución de $I_{d}$ en función del periodo, tenga la misma forma de distribución de $E_{H}$ en función del periodo, característica que 
también se menciona en Rodriguez (2015). Como se comenta más adelante, esta característica ayuda a interpretar resultados para el índice de daño $I_{d}$.

En la evaluación del índice $I_{d}$ en esta investigación se emplearon los mismos valores de los parámetros $\Gamma$ y $H / T$ que intervinieron en el cómputo de $D_{r m}$ aquí descritos; es decir, para $\Gamma$ se empleó el valor 5/4, y los valores de $H / T$ para los casos de edificios en la ciudad de México y en Concepción fueron iguales a $16.2 s^{-1}$ (de la ecuación 5 con $\lambda=5.4 s^{-1}$ y $h=3 m$ ) y $42 s^{-1}$, respectivamente. El parámetro $E_{H}$ se obtuvo de los mismos análisis inelásticos empleados para el cómputo de los espectros $S_{a}$ y $S_{d}$ anteriormente comentados, pero empleando para la fracción de amortiguamiento crítico, $\xi$, el valor 0.02 (Rodriguez, 2015).

La figura 7 muestra resultados del empleo de la ecuación 9 para la evaluación de $I_{d}$ para los casos de los registros en la ciudad de México en 1985 y Concepción en 2010. Con líneas negras continuas, y con líneas grises, se muestran valores calculados de $I_{d}$ para los casos de los terremotos en la ciudad de México y Concepción, respectivamente, para valores de $\mu$ iguales a 2 y 4 . Los resultados para $I_{d}$ siguen la tendencia observada para valores anteriormente comentados de $D_{r m}$ en estos terremotos. En realidad, como se ha comentado, la forma de la distribución de $I_{d}$ en función del periodo, tiene misma forma de distribución de $E_{H}$ en función del periodo. Esto lleva a que en un intervalo de periodos para ambos terremotos, las estructuras más dúctiles tienen índices de daño mayores que los correspondientes a estructuras menos dúctiles, figura 7. Para intervalos de periodos mayores ocurre lo contrario, figura 7. Esta característica para $E_{H}$ es común en los casos de empleo de los registros seleccionados en la ciudad de México en 1985 y Concepción Centro en 2010, lo que se debe a que en ambos casos se tienen suelos con características que se pueden clasificar como deformables, de arcilla en el caso de México, y arenoso en el caso de la estación Concepción Centro.

En el caso del terremoto en la ciudad de México en 1985, los valores encontrados para $I_{d}$ indican colapso de edificios en el intervalo de periodos alrededor de $1.5 s$ a $2.5 s$, ver figura 7, es decir edificios a base de marcos en el intervalo de 8 a 14 niveles, lo que es congruente con la mayor incidencia de colapsos y daños severos observados en edificios de más de 8 niveles, ver figura 2. Los resultados de la figura 7 también son congruentes con la bastante menor incidencia de colapsos y daños observados en edificios de menos de 8 niveles, ver figura 2.

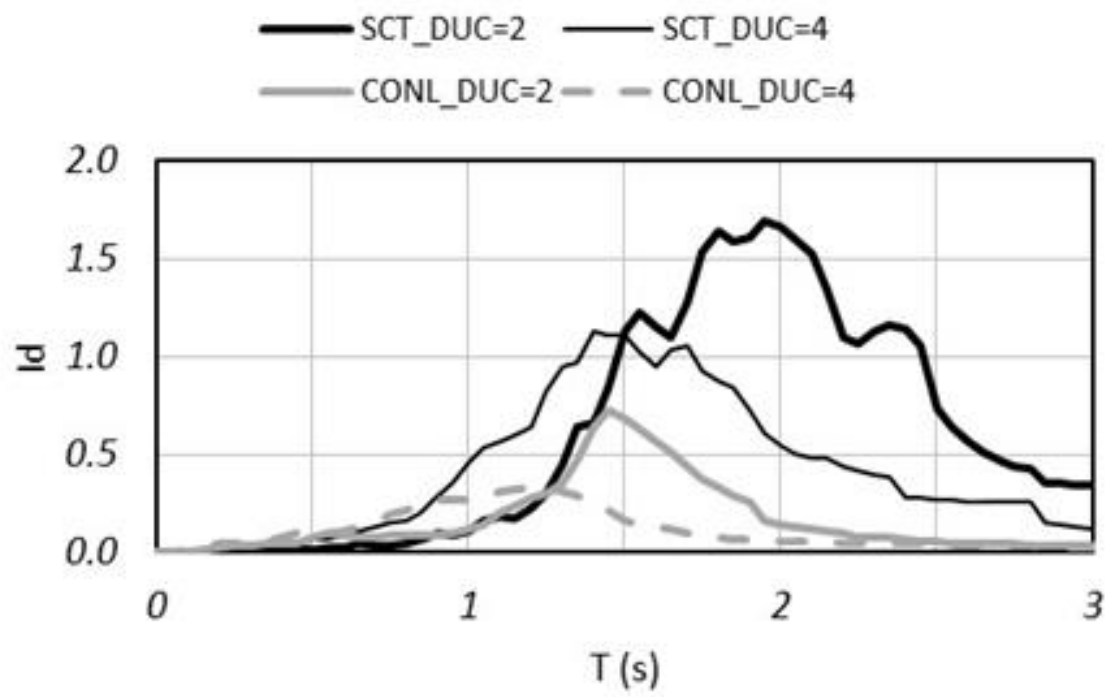

Figura 7. Valores del índice de daño, $I_{d}$, obtenidos con registros de la estación SCT en el terremoto de México 1985, y con registros de la estación Concepción Centro en el terremoto de Maule, Chile 2010 
En el caso del terremoto en la ciudad de México en 1985, los valores encontrados para $I_{d}$ indican colapso de edificios en el intervalo de periodos alrededor de $1.5 \mathrm{~s}$ a $2.5 \mathrm{~s}$, ver figura 7 ; es decir, edificios a base de marcos en el intervalo de 8 a 14 niveles, lo que es congruente con la mayor incidencia de colapsos y daños severos observados en edificios de más de 8 niveles, ver figura 2. Los resultados de la figura 7 también son congruentes con la bastante menor incidencia de colapsos y daños observados en edificios de menos de 8 niveles, ver figura 2.

En el caso de edificios en Concepción en el terremoto de 2010, la figura 7 indica que los valores máximos encontrados para $I_{d}$ para las ductilidades dos y cuatro son menores que 1 , es decir no indican colapso de edificios, aunque $I_{d}$ se acerca a 1 en un muy pequeño intervalo de periodos para el caso $\mu=2$. El índice $I_{d}$ alcanza valores máximos en el intervalo de periodos de $1 s$ a $1.8 \mathrm{~s}$.

\section{CONCLUSIONES}

1. Los valores de demandas espectrales de desplazamientos, calculadas con registros de aceleraciones en los terremotos que se estudiaron en esta investigación, en todos los casos fueron mayores que las especificadas por las normativas de los diversos lugares que experimentaron estos terremotos, con valores de demandas que en algunos casos excedieron a las especificadas en alrededor de $100 \%$. Los daños o colapsos en edificaciones en los terremotos estudiados no fueron esperados por los ingenieros que diseñaron estas edificaciones. Esto muestra las considerables incertidumbres en los procedimientos de diseño por sismo de normativas de construcción. Esto sugiere que las normativas de construcción, además de considerar la resistencia lateral de estructuras, deben hacer énfasis en un control adecuado de desplazamientos en la respuesta inelástica de la estructura durante un terremoto, así como especificar un detallado del refuerzo que permita esta respuesta. Además, las hipótesis empleadas para definir las acciones sísmicas de diseño merecen ser revisadas.

2. A pesar de lo anterior, las normativas de diseño de estructuras por sismo en México, existentes sólo para algunas regiones del país, hacen excesivo énfasis en las fuerzas sísmicas de diseño, y le dan menos importancia al control de desplazamientos en la estructura y al detallado del refuerzo en ella. Esto ha llevado a que los ingenieros en la práctica en muchos casos no tomen en cuenta la importancia del control de daños estructurales y no estructurales por sismo. Para este control es relevante la selección de un sistema sismo resistente adecuado, lo cual en un intervalo amplio del periodo fundamental de edificios no es posible con el empleo de marcos, como se ha mostrado en esta investigación.

3. El uso de marcos es muy común en México, en gran parte por ser preferidos por los arquitectos, quienes generalmente en su solución arquitectónica inicial proponen principalmente el empleo de columnas, sin considerar la utilización de muros estructurales. Esta situación se agrava porque las normativas existentes en el país, a diferencia de las de otros países en zonas sísmicas, permiten de manera equivocada el empleo de estructuras de ductilidad limitada (factor de comportamiento sísmico de dos o menos) en zonas de alta sismicidad, como por ejemplo la zona de lago del DF o zonas en la costa del Pacífico. Como se ha mostrado en esta investigación, contar con sólo resistencia en la estructura sin capacidad de deformación lateral no es suficiente, ya que como se ha observado en varios terremotos en el mundo, incluyendo México, las demandas en terremotos seguramente excederán a las de diseño, incluso en una vida útil mucho menor que la esperada, como se observó en Nueva Zelanda en los terremotos de 2010 y 2011. En estos casos, el contar 
con una reserva de capacidad de deformación lateral, puede significar el evitar el colapso de la estructura en un terremoto y por tanto la pérdida de vidas humanas.

4. Las observaciones anteriores sugieren la necesidad de cambios importantes en la práctica de diseño sísmico en México, para lo cual se recomienda que normativas de construcción, en sus requisitos para seleccionar un sistema estructural sismo resistente (por ejemplo a base de marcos o a base de muros), incentiven el empleo de sistemas sismo resistentes con muros estructurales, con un detallado de refuerzo adecuado. Este detallado podría ser el especificado por la norma ACI 318-14 (2014) en su parte de diseño por sismo, que se basa en la experiencia obtenida de la observación del comportamiento de edificios con muros estructurales de CR en el terremoto de Maule, Chile, 2010. Además, se recomienda que las normativas de diseño por sismo en México, como primer paso del diseño, especifiquen de manera explícita el empleo de espectros de desplazamientos, limitando de manera realista los valores permisibles de distorsiones en edificios para estos espectros.

5. Se debe tener en cuenta que la ductilidad en la estructura no lo es todo, no debe ser el objetivo final de diseño sísmico, ya que implica daño en la estructura. En los casos en que después de un terremoto sea necesario el poder contar con el funcionamiento total de una estructura, como es por ejemplo el caso de hospitales, se deben usar sistemas estructurales innovadores, como son por ejemplo los a base de empleo de aisladores en la base de la estructura. Esta práctica no existe en México, y ya se utiliza en el mundo, incluyendo países en Latinoamérica como Chile desde hace varios años, o recientemente Perú. Para ello es necesario que exista en México normativas, o al menos recomendaciones, de diseño sísmico de estructuras con aisladores en su base.

\section{AGRADECIMIENTOS}

Se agradece al Profesor Patricio Bonelli de la Universidad Técnica F. Santa María en Valparaíso, Chile, por su cuidadosa lectura crítica del manuscrito, que contribuyó a mejorarlo, y por su generosidad en proporcionar información de temas relacionados con el terremoto de Maule, 2010, que sirvieron para esta investigación. Se agradece a los revisores anónimos y al Dr. S K Singh, sus comentarios al manuscrito, lo que permitió mejorarlo.

\section{REFERENCIAS}

ACI Committee 318-14 (ACI 318, 2014), "Building Code Requirements for Reinforced Concrete (ACI 318-14)", American Concrete Institute, Farmington Hills, MI.

ASCE/SEI 7-10 (2010), "Minimum Design Loads for Buildings and Other Structures", American Society of Civil Engineers, Estados Unidos.

ASCE/SEI 7-16 (2017), "Minimum Design Loads for Buildings and Other Structures”, American Society of Civil Engineers, Estados Unidos.

Bonelli, P (2015), Comunicación personal.

Boroschek, R, V Contreras, D Y Kwak y P Stewart (2012), "Strong Ground Motion Attributes of the 2010 Mw 8.8 Maule, Chile, Earthquake", Earthquake Spectra, Vol. 28, No. S1, pp. S19-S38. DOI: 10.1193/1.4000045. 
Bradley, B, M Quigley, R Van Dissen y Litchfiled N (2014), "Ground Motion and Seismic Source Aspects of the Canterbury Earthquake Sequence", Earthquake Spectra, Vol. 30, No. 1, pp. 1-15. DOI: 10.1193/030113EQS060M.

Carr, A (2011), "RUAUMOKO, Computer Program Library", University of Canterbury, Department of Civil Engineering.

Comisión Federal de Electricidad (2008), "Manual de diseño de obras civiles por Sismo", México, DF.

EERI (2011), “The M 6.3 Christchurch, New Zealand, Earthquake of February 22, 2011”, EERI Special Earthquake Report, pp. 1-16.

Elwood, K J y M O Eberhard (2009), "Effective Stiffness of Reinforced Concrete Columns", ACI Structural Journal, Julio-Agosto, pp. 476-484.

Gerstenberger, M, G McVerry, D Rhoades y M Stirling (2014), "Seismic Hazard Modeling for the Recovery of Christchurch", Earthquake Spectra, Vol. 30, No. 1, pp. 17-29. DOI: 10.1193/021913EQS037M.

Ghosh, S K (2016), “Alternative Diaphragm Seismic Design Force Level of ASCE 7-16," Structure magazine, Marzo, pp. 18-22.

Guendelman, T, G R Saragoni y R Verdugo (2012), "Chilean Emergency Seismic Design Code for Buildings After El Maule 2010 Earthquake”, Proc. 15 WCEE, paper 4480, Lisboa.

Instituto Nacional de Normalizacion (1996), "Norma Chilena Oficial. Diseño Sísmico de edificios", NCh433.Of 1996, Chile.

Lagos, R, M Kupper, J Lindenberg, P Bonelli, R Saragoni, T Gueldelman, L Massone, R Boroschek y F Yanez (2012), "Seismic Performance of High-Rise Concrete Buildings in Chile", International Journal of High-Rise Buildings, Vol. 1, No. 3, pp. 181-194.

Martinelli, P y F Filippou (2009), "Simulation of the shaking table test of a seven-story shear wall building”, Earthquake Engineering \& Structural Dynamics, Vol. 38, pp. 587-607. DOI: 10.1002/eqe.897.

Massone, L, P Bonelli, R Lagos, C Luders, J Moehle y J Wallace (2012), "Seismic Design and Construction Practices for RC Structural Wall Buildings", Earthquake Spectra, Vol. 28, No. S1, pp. S245-S256. DOI: 10.1193/1.4000046.

Noreña, E, C Castañeda y J Iglesias (1989), “The Mexico Earthquake of September 19, 1985- Evaluation of the Seismic Capacity of Buildings in Mexico City", Earthquake Spectra, Vol. 5, No. 1, pp. 1924. DOI: $10.1193 / 1.1585508$.

Panagiotou, M (2008), "Seismic Design, Testing, and Analysis of Reinforced Concrete Wall Buildings", Tesis Doctoral, University of California, San Diego, USA, supervisada por J. Restrepo.

RCDF (1976), “Reglamento de Construcciones para el DF”. Diario Oficial de la Federación.

Rodriguez, M E y J C Aristizabal (1999), "Evaluation of a Seismic Damage Parameter", Earthquake Engineering \& Structural Dynamics, Vol. 28, pp. 463-477. DOI: 10.1002/(SICI)10969845(199905)28:5<463::AID-EQE818>3.0.CO;2-V.

Rodriguez, M E y M Torres (2013), "Seismic Behavior of a Type of Welded Precast Beam-Column Connection," Prestresed Concrete Institute Journal, Summer Issue, USA.

Rodriguez, M E (2015), "Evaluation of a proposed damage index for a set of earthquakes", Earthquake Engineering \& Structural Dynamics, Vol. 44, pp. 1255-1270. DOI: 10.1002/eqe.2512. 
Saiidi, M y M Sozen (1981), "Simple nonlinear analysis of RC structures", Journal of Structural Engineering, ASCE, Vol. 197, pp. 937-952.

Shimazaki, K y M A Sozen (1984), "Seismic Drift of Reinforced Concrete Structures", Technical Report, Hazama-Gumi, Tokyo, pp. 145-165.

Sozen M (1997), "Drift-driven design for earthquake resistance of reinforced concrete", EERC-CUREe Symposium in Honor of Vitelmo Bertero, Report UCB/EERC-97/05, EERC, University of California, Berkeley.

Standards New Zealand (2004), NZS 1170.5:2004, "New Zealand Standard. Structural design actions. Part 5: Earthquake actions-New Zealand".

Wallace, J, L Massone, P Bonelli, J Dragovich, R Lagos, C Luders y J Moehle (2012), "Damage and Implications for Seismic Design of RC Structural Wall Buildings", Earthquake Spectra, Vol. 28, No. S1, pp. S281-S299. DOI: 10.1193/1.4000047.

Wang, Z (2015), "Predicting or Forecasting Earthquakes and the Resulting Ground-Motions Hazards: A Dilemma for Earth Scientists", Seismological Research Letters, Vol. 86, No. 8, pp. 1-5. DOI: $\underline{10.1785 / 0220140211 .}$ 